Rheo-optical Analysis of Functionalized Graphene Suspensions

Peer-reviewed author version

REDDY, Naveen; Natale, Giovanniantonio; Prud'homme, Robert \& Vermant, Jan (2018) Rheo-optical Analysis of Functionalized Graphene Suspensions. In:

Langmuir, 34 (26), p. 8744-8751.

DOI: 10.1021/acs.langmuir.8b01574

Handle: http://hdl.handle.net/1942/26215 


\title{
Rheo-optical Analysis of Functionalised Graphene Suspensions
}

\author{
Naveen K Reddy, ${ }^{*, \dagger}$, Giovanniantonio Natale, ${ }^{\Uparrow}$ Robert K Prudhomme, ${ }^{\S}$ and Jan \\ Vermant $\|$ \\ $\dagger$ Hasselt University, Martelarenlaan 42, 3500 Hasselt, Belgium \\ $\ddagger I M O-I M O M E C$, Wetenschapspark 1, 3590 Diepenbeek, Belgium \\ IDepartment of Chemical and Petroleum Engineering, University of Calgary, 2500 \\ University Dr. NW, Calgary, AB T2N 1N4, Canada \\ $\S$ Department of Chemical Engineering, Princeton University, Princeton, New Jersey \\ 08544, USA \\ ||Department of Materials, ETH Zrich, Vladimir-Prelog-Weg 5, 8093 Zrich, Switzerland \\ E-mail: naveen.reddy@uhasselt.be
}

\begin{abstract}
Wet processing of graphene flakes is an important route for creating novel materials. In the present work flow dichroism and small angle light scattering are used to investigate the state of functionalized graphene flakes in suspension and the response to shear flow. In line with expectations from scaling theory, the functionalized graphene sheets are present as flat objects, and flow increases the orientation as for hard spheroidal objects. Comparing the flow induced orientation of thick gold decahedra with the thin graphene flakes shows essentially the same behaviour, except for effects of polydispersity. Adequate prediction of the effects of flow on orientation of graphene flakes is important for designing wet processed graphene based composite materials. In this work we show that quantitative prediction is now possible.
\end{abstract}




\section{Introduction}

Graphene sheets are two-dimensional (2D) materials composed of carbon atoms arranged in a honeycomb structure. ${ }^{1}$ Pristine graphene sheets are known to own outstanding mechanical and electrical properties; a Young's modulus of $1 \mathrm{TPa}$, making it one of the strongest material; thermal conductivity of $5000 \mathrm{~W} / \mathrm{m}^{3} \mathrm{~K}$, only comparable to that of single walled carbon nanotubes. ${ }^{2-5}$ Due to their $2 \mathrm{D}$ nature, it is relatively easy to produce macroscopic films and sheets that can be used in diverse applications such as large area conducting surfaces, ${ }^{9}$ interface stabilised emulsions, ${ }^{10}$ transparent electrodes, ${ }^{11}$ drug sensors ${ }^{12}$ and nanocomposites. ${ }^{5}$ In all of the above applications, graphene sheets are dispersed in a medium to be then deposited or transferred to a substrate. This dispersion processing route is generally followed since it is efficient, inexpensive, and can process large quantities. Once dispersed in solution, a precise knowledge of the orientation and ordering of graphene sheets during flow is required to control the final macroscopic properties of the produced materials. Rheological properties evidently play an important role in controlling the structure and orientation of the grapheneous materials. ${ }^{6}$ For graphene oxide a pronounced shear-induced alignment has been reported, which revealed a surprising interplay between shape and single layer flexibility effects. ${ }^{7}$ However, due to the many defects graphene oxide is super-flexible when compared to Graphene. The bending modulus of graphene is of order $100 \mathrm{k}_{\mathrm{B}} \mathrm{T}^{6,8}$ and it is interesting to investigate how graphene orients under flow and if, unlike its oxidised counterpart, can be treated as a rigid sheet, to understand and predict how processing can be done. In this article, we experimentally and theoretically, determine the flow-induced anisotropy of functionalized graphene sheets in suspension.

To this end, rheo-optical measurements will be used; as early as 1973 Mason and coworkers used turbidity measurements to obtain quantitative information on the motion of particles and their alignment with respect to the flow direction. ${ }^{13}$ However, turbidity alone does not allow one to measure both the dichroism and orientation angle of the distribu- 
tion; moreover turbidity requires rather concentrated systems. The draw backs of turbidity measurements were overcome by using flow dichroism. ${ }^{14,15}$ Flow dichroism has the ability to elucidate the nature of particle motion and average degree of alignment simultaneously, and can be used even for nanoparticles. ${ }^{16-20}$ Flow dichroism has been used in the past to study both rod-like and disk-like nanoparticles in suspension. ${ }^{14,21-24}$ In all the cases where disk-like nanoparticles are studied the particles were made of high bending modulus materials such as clay or gold. ${ }^{14,19,25}$ Using flow dichroism and static light scattering, we study the behaviour of functionalized graphene sheets (FGS) to investigate their 2D nature in suspension. Graphene oxide sheets have been suggested to exist in a crumpled state ${ }^{26,27}$ but more precise experiments only found relatively flat configurations. ${ }^{28}$ Scaling arguments from polymer theory for 2D polymers suggest that self-avoiding surfaces make the "flat" state dominant for $2 \mathrm{D}$ polymers, even for very small values of bending rigidity. The local bending is important in the self-avoiding nature and this leads to flatness. ${ }^{29}$ Yet some fluctuations, both parallel and perpendicular to the sheet are going to be present. For graphene oxide particles in concentrated suspensions it was observed that they become even flatter when sheared. ${ }^{7}$

The knowledge obtained on the state of FGS in suspension will eventually lead to a better control over the solution process and consequentially, to a superior product design. Depending on the final application, flow-induced alignment of the constitutive elements can be an advantage or disadvantage. On one hand, flow-induced alignment is a disadvantage for high strength and high electrical conductivities because it reduces the density of contact junctions per unit volume which leads to a lower strength and electrical conductivity. ${ }^{5,30}$ On the other hand, alignment of particles can be used as a means to create materials with anisotropic properties. To our knowledge, no experimental work has been carried out on the flow-induced anisotropy of functionalized graphene sheet suspensions in the dilute regime. 
Rheo-optical techniques provide unique information linking rheological measurements with in-situ microstructural events in real time. The material properties of interest is the refractive index tensor, $\mathbf{n}=\mathbf{n}^{\prime}-i \mathbf{n}^{\prime \prime}$. Dichroism is related to differences in the principal eigenvalues of the imaginary part of the refractive index tensor, $\Delta n^{\prime \prime}$. Physically, dichroism is related to the attenuation in the transmitted light generally due to absorption and scattering. Flow-induced dichroism of both single-walled and multi-walled carbon nanotubes have been experimentally determined by Fry et al. ${ }^{31,32}$ They showed that the concentration-scaled dichroism, which directly relates to the order parameter, scales as $\mathrm{Pe}^{2.0}$ at low $\mathrm{Pe}$ and $\mathrm{Pe}^{0.25}$ for modest Pe, where Pe is the Péclet number. Fry et al. used a parallel plate setup to measure the flow alignment of CNTs allowing them to access only gradient direction and therefore they could not measure rotational diffusion of CNTs experimentally. ${ }^{31,32} \mathrm{~A}$ more interesting and useful direction to measure flow induced alignment is the vorticity direction, which could be accessed by using a Couette cell. ${ }^{14,17,33}$ To this end, Natale et al. ${ }^{20}$ have recently reported results of flow induced alignment of multi-walled CNTs using Couette cell. Here by probing in the vorticity direction they have been able to experimentally obtain rotational diffusion (Dr) using flow relaxation experiments. The scaling relation $\mathrm{Pe}^{0.25}$ obtained for CNTs agrees qualitatively with the Doi and Edwards prediction for semiflexible polymers. ${ }^{34}$

Static light scattering (SLS) is a powerful technique that provides information related to the shape of the particles (when probed in the right scattering vector range). For disk like particles, the scattering vector $(q)$ between $q=2 \pi / d$ to $q=2 \pi / t$ in the reciprocal space, where $d$ is the diameter of the disk and $t$ the thickness, provides information on fractal nature of disks surface. Power law relationship between scattered intensity and $q$ with exponent of 2.0 within the correct $q$ range indicates that the particle is a flat disk. If the sheets are crumpled then this exponent is expected to be around 2.5. SLS has been used in the past to study graphite oxide suspensions where the authors found that the particles remained flat 
without crumpling, ${ }^{27,28}$ in agreement with recent scaling argument for $2 \mathrm{D}$ polymers ${ }^{29}$

In the present work, flow dichroism and static light scattering techniques are used to shed light on the state of FGS in suspension and on their orientation dynamics. In addition, the extent of FGS alignment obtained from flow dichroism measurements is compared to the experimental data of oblate (decahedron) gold nanoparticles to highlight differences in the orientation behaviour induced by the different geometrical shapes of these two nanoparticles. ${ }^{19}$ The orientation evolution of these dilute systems is also analysed by modelling the orientation distribution function as a convected quantity. ${ }^{35}$ The Fokker-Planck or Smoluchowski equation is used to describe the orientation state of the systems. The particles are modelled as Brownian spheroids following the dynamics described by Jeffery's equation. ${ }^{36,37}$ Rotational diffusion obtained from flow relaxation experiments and the nanoparticles' form factor obtained from transmission electron microscopy (TEM) and atomic force microscopy (AFM) are used as input to the theory. A thin disk geometry is used to represent the shape of graphene while an oblate spheroid, for gold nanoparticles (decahedron). The theoretical description, without the use of any fitting parameters, can overall predict the flow alignment obtained from dichroism experiments for these two systems. For the FGS suspension, polydispersity is also included in the model. Hence, results from dichroism and light scattering indicates FGS in suspension are not crumpled and their orientation behaviour overall follows the behaviour of rigid particles. To complement flow dichroism experiments, we perform static light scattering experiments which confirms FGS in suspension to be flat objects, in agreement with scaling arguments ${ }^{29}$ 


\section{Experimental}

\section{Materials}

Details of FGS synthesis with their corresponding microscopy images was reported by McAllister et al. ${ }^{38}$ and the synthesis and characterisation of the gold dodecahedra is given by Reddy et al. ${ }^{19}$ The average thickness and diameter of FGS is around $1.75 \mathrm{~nm}$ and $500 \mathrm{~nm}$ respectively and contains functional epoxy and hydroxyl groups. ${ }^{39}$ The thickness of graphene particles resulted to be uniform while large polydispersity was encountered in term of lateral dimensions, see Fig. 1. FGS was diluted using 100 mPas mineral oil (Sigma), which was Newtonian for all the range of shear rate analysed. Four different concentrations of graphene sheets in the hydrodynamically dilute regime were studied in this work and they are listed in Table 1. For comparison, two different dimensions of gold decahedrons in the hydrodynamically dilute regime were used; Length/diameter $=170 / 105 \mathrm{~nm}$ and 292/180 nm. Oblate gold spheroids in water were diluted with glycerol/water mixture. In both cases the viscosity of the suspending fluid was chosen such that the hydrodynamically dominated regime was accessible in the limited shear rate window of the rheometer.

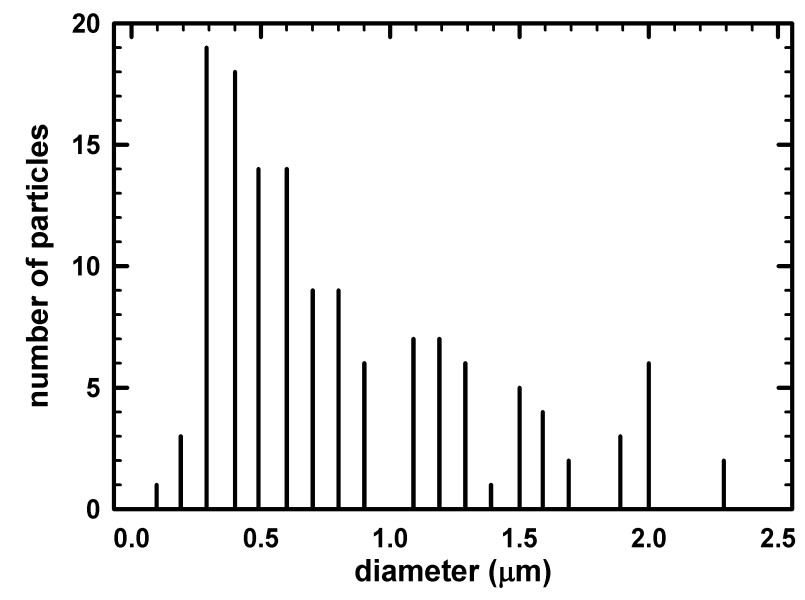

Figure 1: Plot of number of particles as a function of FGS diameter. 
Table 1: Concentrations of graphene suspensions

\begin{tabular}{ll}
\hline Name & wt/vol. \% \\
\hline Sample-1 & $3.00 \times 10^{-4}$ \\
Sample-2 & $6.07 \times 10^{-4}$ \\
Sample-3 & $1.21 \times 10^{-3}$ \\
Sample-4 & $2.43 \times 10^{-3}$ \\
Sample-5 & $3.04 \times 10^{-3}$ \\
\hline
\end{tabular}

\section{Rheo-optics}

Dichroism measurements are performed using an in-house rheo-optical setup based on an MCR300 stress-controlled rheometer as the mechanical platform (Paar Physica, Austria). ${ }^{40}$ Here a Couette cell is used with a laser light $(10 \mathrm{~mW}$ He-Ne, $\lambda=632.8 \mathrm{~nm})$ being sent along the vorticity direction of the flow cell. This allows us to determine the flow-induced dichroism and orientation angle simultaneously as a function of the applied shear rate. The inner and outer radii of the Couette cell are 16.95 and $17.95 \mathrm{~mm}\left(\mathrm{R}_{i} / \mathrm{R}_{o}=0.94\right)$ respectively and the optical path length is $21 \mathrm{~mm}$. All experiments are performed at room temperature. The optical train consists of a laser $(\lambda=632.8 \mathrm{~nm})$, followed by a Glan-Thompson polarizer (P1, Newport, USA), a photoelastic modulator (PEM, Beaglehole Instruments, NZL) oriented at $45^{\circ}$ with respect to $\mathrm{P} 1$ and a zero-order quarter-wave plate (Newport) at $0^{\circ}$ with respect to $\mathrm{P} 1$. Using a combination of prisms the beam is passed into the Couette cell in the vorticity direction. The transmitted light is collected by a photodiode. Data from the photodiode is sent through a low-pass filter into two Lock-In amplifiers (Stanford Research Systems Model 830) from which we obtain the first and second harmonic signals $\left(R_{1}\right.$ and $\left.R_{2}\right)$. The setup is calibrated in such a manner that the orientation in the flow direction corresponds to a positive dichroism. The extinction coefficient $\left(\delta^{\prime \prime}\right)$ and the orientation angle $(\chi)$ are obtained from the first and second harmonics $\left(R_{1}\right.$ and $\left.R_{2}\right)$ along with the calibration values for $\mathrm{J}_{1}(\mathrm{~A})$ and $\mathrm{J}_{2}(\mathrm{~A})$ (Bessel functions of the first kind) using Eq. 1 and $3 .{ }^{33}$ The associated dichroism $\left(\Delta n^{\prime \prime}\right)$ is obtained from Eq. 2 


$$
\delta^{\prime \prime}=-\operatorname{sgn}\left(R_{2}\right) \tanh ^{-1}\left\{\frac{1}{2} \sqrt{\frac{R_{1}}{-\mathrm{J}_{1}(A)}+\frac{R_{2}}{-\mathrm{J}_{2}(A)}}\right\}
$$

$$
\Delta n^{\prime \prime}=\frac{\delta^{\prime \prime} \lambda}{2 \pi d}
$$

where $d$ is the optical path length and $\lambda$ the wavelength of the laser light source. The orientation angle is obtained from:

$$
\chi=\frac{1}{2} \tan ^{-1}\left\{\frac{R_{1} / \mathrm{J}_{1}(A)}{R_{2} / \mathrm{J}_{2}(A)}\right\}
$$

\section{Static light scattering}

Static light scattering (SLS) measurements are performed on an ALV CGS-3 (Langen, Germany) compact goniometer system equipped with a multi-tau digital correlator (ALV/LSE5003). The light source is a $35 \mathrm{~mW}$ He-Ne laser with a wavelength $(\lambda)$ of $632.8 \mathrm{~nm}$. Samples are equilibrated at the measuring temperature of $20^{\circ} \mathrm{C}$ for 30 minutes prior to any measurement. A circulating water bath is used to control the temperature within $\pm 0.1{ }^{\circ} \mathrm{C}$ of the set value. SLS measurements are performed from scattering angles of 30 to $120^{\circ}$ which corresponds to $6.835 \times 10^{-3} \mathrm{~nm}^{-1}<\mathrm{q}<2.28 \times 10^{-2} \mathrm{~nm}^{-1}$, where $q$ is the scattering vector given by $q=4 \pi n \sin (\theta / 2) / \lambda$, with $n=1.47$ the refractive index of the suspending medium and $\theta$ the scattering angle. 


\section{Results and discussion}

\section{Static light scattering}

We performed static light scattering (SLS) experiments on dilute suspensions to investigate if FGS is indeed present as a flat 2D structure in suspension, even in the absence of flow. In a light scattering experiment, particles act as point scatterers, when $q d<<1$, whereas for $q d>>1$ the internal surface of the particle is probed, where $d$ is the characteristic size of the particle and $q$ the wave vector. In the region $q d>>1$ the scattered intensity $I_{s}$ shows a power law dependency $I(q) \propto q^{-d_{f}}$, where $d_{f}$ is the fractal dimension of the particle. Flat $2 \mathrm{D}$ sheets show a fractal dimension $d_{f}=2.0$ in the range $q=2 \pi / d=7 \times 10^{6} \mathrm{~m}^{-1}$ to $q=2 \pi / t=3.6 \times 10^{9}$ $\mathrm{m}^{-1}$, where $d$ in the case of FGS is the diameter and $t$ the thickness. The fractal dimension for a compact/collapsed particle is $d_{f}=3.0 .{ }^{26,27}$ An average fractal dimension of $d_{f}=2.1$ is obtained, which indicates essentially flat surfaces (with some wrinkles). Although the data only covers a decade in $q$, it is consistent with data on the even more flexible graphite oxide flakes which also does not show a crumpled state in suspension, but rather flat sheets, ${ }^{28}$ and in line with the expected morphology based on scaling arguments. ${ }^{29}$

\section{Steady state dichroism}

The flow-induced dichroism and the average orientation angle of the functionalized graphene sheet suspensions as a function of applied shear rate and concentration are shown in Fig. 3. At rest, due to Brownian motion, non-spherical nanoparticles are randomly oriented resulting in negligible dichroism with an isotropic orientation angle of $45^{\circ}$. When a shear flow is applied to a randomly oriented sample, the flow on an average aligns the particles (for a polydisperse system), causing the flow dichroism to increase and the orientation angle to decrease. Once the particles are flow aligned the dichroism reaches a maximum value and any further increase in the magnitude of the shear rate will not affect the magnitude of the dichroism to a significant extent. In this high shear rate regime, the optical response 


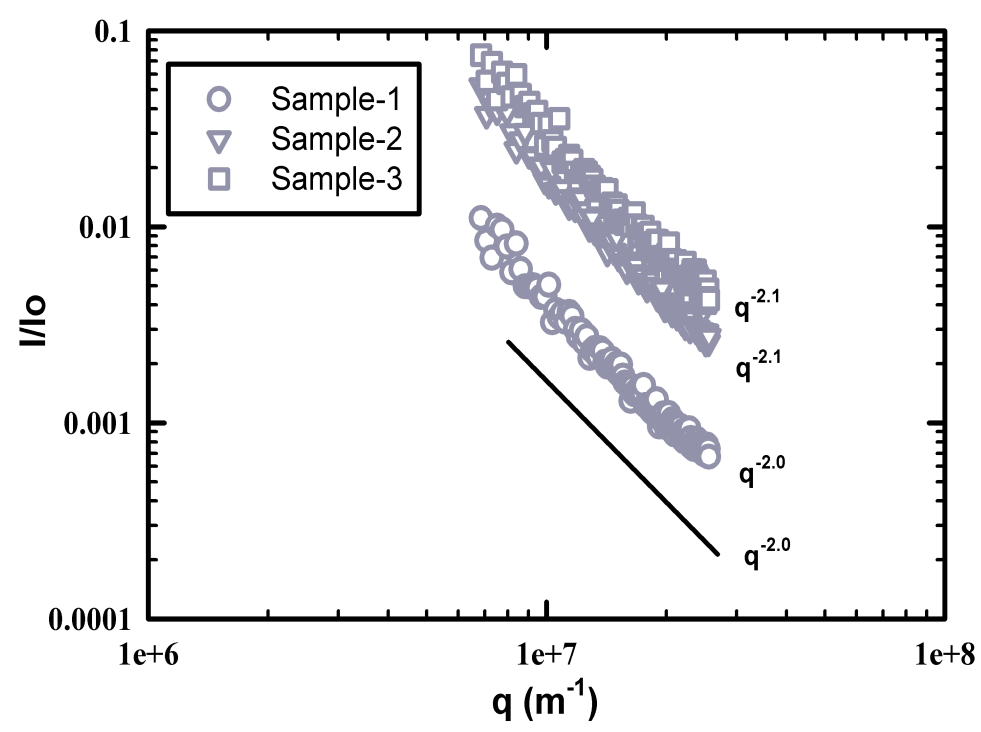

Figure 2: Scattered light intensity normalised by incident light obtained from static light scattering is plotted as a function of scattering vector $(q)$ for 3 different FGS suspensions. The solid line represents the $q^{2}$ dependence. The $q$ dependence for each FGS concentration is shown next to there respective curve.

is completely dominated by hydrodynamic effects as shown in Fig. 3.a. where dichroism increases linearly with concentration, at all shear rates. The scattering intensity for all shear rate decreases linearly with increasing in concentration as shown in the inset of Fig. 3.b. This linear dependence of both flow dichroism and scattering intensity indicates that the FGS concentrations used are in the hydrodynamically dilute regime. The average orientation angle depends only on the orientation of particles in the suspension with respect to the flow direction and therefore is independent of the concentration of particles, see Fig. 3.b. To compare the flow behaviour of a flat disk-like FGS suspension to that of oblate spheroids, we performed dichroism experiments using oblate gold nanoparticles as shown in Fig. 7. From plots of orientation angle as a function of shear rate for both between FGS and oblate gold nanoparticles it can be seen that at low shear rates orientation angle of gold nanoparticles is close to isotropic state whereas for FGS it is already low at around $20-25^{\circ}$. At intermediate shear rates the slope of the orientation angle for FGS is around 0.25 and for gold nanoparticles its round 0.9. At high shear rates gold nanoparticles are completely flow 
aligned with orientation angle close to $0^{\circ}$ whereas for FGS its around $5^{\circ}$. To explain the above differences we need to look at their transient flow behaviour, that is dealt in the next section.
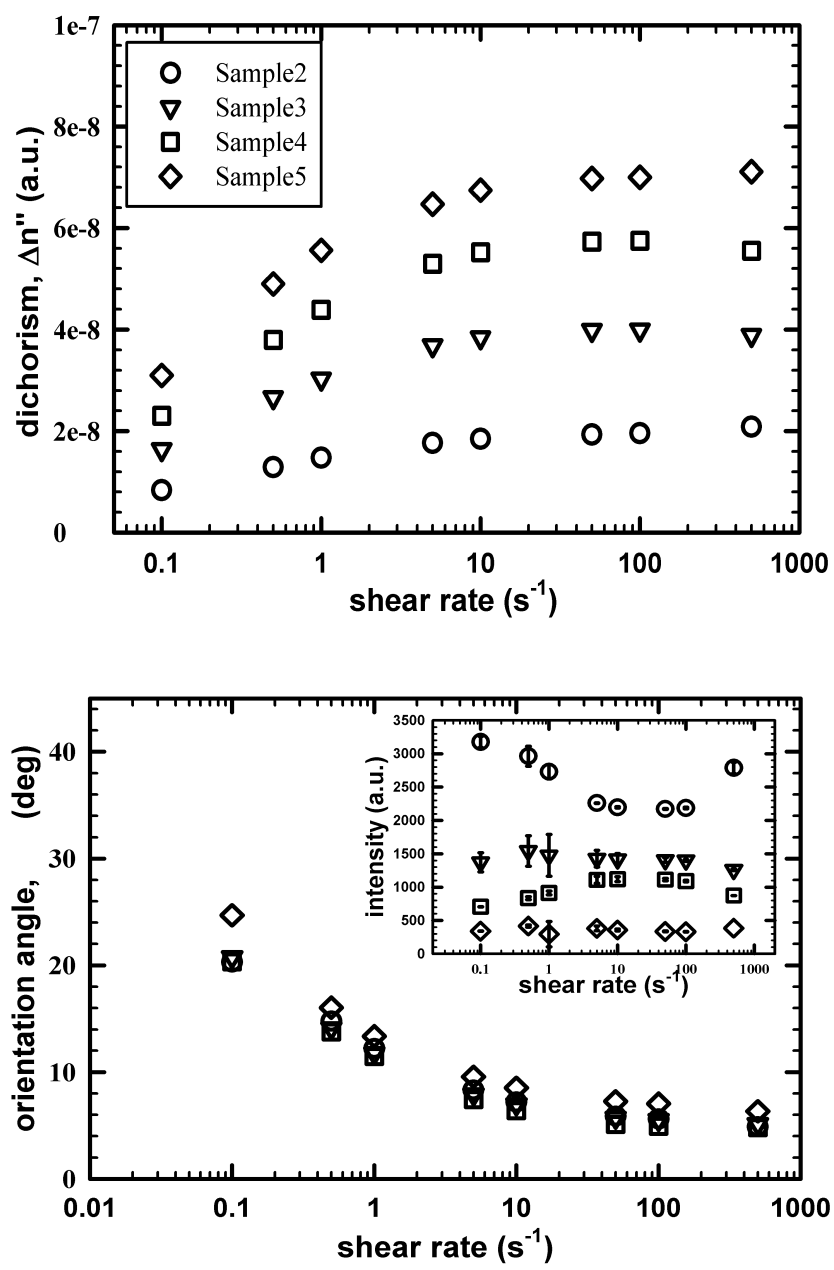

Figure 3: a) Flow dichroism as a function of shear rate for four different concentrations of FGS in the dilute regime and $b$ ) evolution of the average orientation angle with respect to flow direction as a function of shear rate. The inset in Fig. b) shows the intensity of the transmitted light through the four suspensions whose concentration is given in Table 1.

\section{Relaxation experiments}

Flow cessation experiments in the hydrodynamic regime (large Peclet number regime) are used to obtain the rotational diffusion coefficient $\left(\mathrm{D}_{r}\right)$ from dichroism relaxation. After 


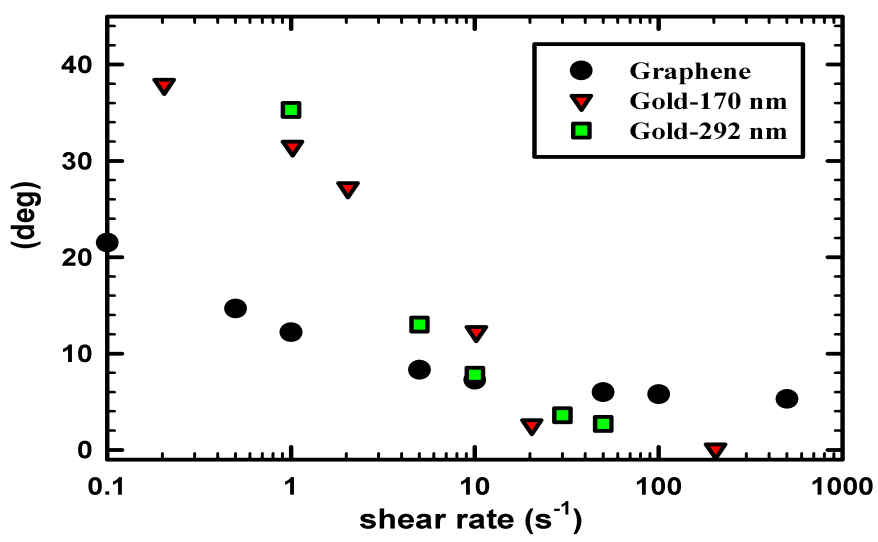

Figure 4: Orientation angle as a function of shear rate for oblate gold nanoparticles with diameters of 170 and $292 \mathrm{~nm}$. For comparison average orientation angle for FGS from 3.b is also shown.

cessation of flow, particles that are oriented along the flow direction relax back to the isotropic state by Brownian motion. The decay in dichroism due to particle relaxation is used to obtain $\mathrm{D}_{r} \cdot{ }^{41}$ For mono-dispersed particles the dichroism relaxation shows a single exponential, Eq. 4 with $\beta=1 .{ }^{42}$

$$
f(t)=e^{-\left\{\frac{t}{\tau}\right\}^{\beta}}
$$

where $t$ is time and $\tau=\left(6 D_{r}\right)^{-1}$. If the particles are polydisperse, the relaxation will be a stretched exponential (Eq. 4) with $0<\beta<1$. $^{42}$ Dichroism relaxation upon flow cessation for oblate gold spheroids with $\mathrm{L}=170 \mathrm{~nm}$ and $292 \mathrm{~nm}$ is shown in Fig. 5. The relaxation curves for both particles can be fitted with a single exponential to obtain rotational diffusion coefficients of $0.23 \mathrm{~s}^{-1}$ (for $\mathrm{L}=170 \mathrm{~nm}$ ) and $0.14 \mathrm{~s}^{-1}$ (for $\mathrm{L}=292 \mathrm{~nm}$ ). The single exponential fits to the oblate gold spheroids indicate that the these particles are relatively monodisperse, in agreement with earlier results. ${ }^{19}$ Using the experimentally obtained value for $\mathrm{D}_{r}$, characteristic sizes of the particles are obtained from Eq. 5 for oblate spheroids. ${ }^{43}$

$$
D_{r}=\left(\frac{k_{\mathrm{B}} T}{4 \eta_{o} V}\right)\left(\frac{r^{2}}{r^{4}-1}\right)\left\{\frac{r^{2}-2}{\sqrt{r^{2}-1}} \tan ^{-1}\left(\sqrt{r^{2}-1}\right)+1\right\} \text { for } r>1
$$


where $\mathrm{k}_{\mathrm{B}}$ is the Boltzmann constant, $T$ the temperature, $\eta_{o}$ the viscosity of the solvent, $r$ $=\mathrm{b} / \mathrm{a}$ the ratio of semi-major axis to the semi-minor axis and $V$ the volume $\left(4 \pi a b^{2} / 3\right)$. In Eq. 5 the effective aspect ratio for oblate gold spheroids is taken to be 0.55 , obtained from flow start up experiments. ${ }^{19}$ The characteristic size calculated for oblate gold spheroids from Eq. 5 was $197 \mathrm{~nm}$ and $235 \mathrm{~nm}$, which is in good agreement with TEM measurements of $170 \mathrm{~nm}$ and $292 \mathrm{~nm}$. The small difference of $15-20 \%$ can be attributed to the fact that flow dichroism measurements average over a large amount of particles as compared to few hundred particles in TEM measurements.

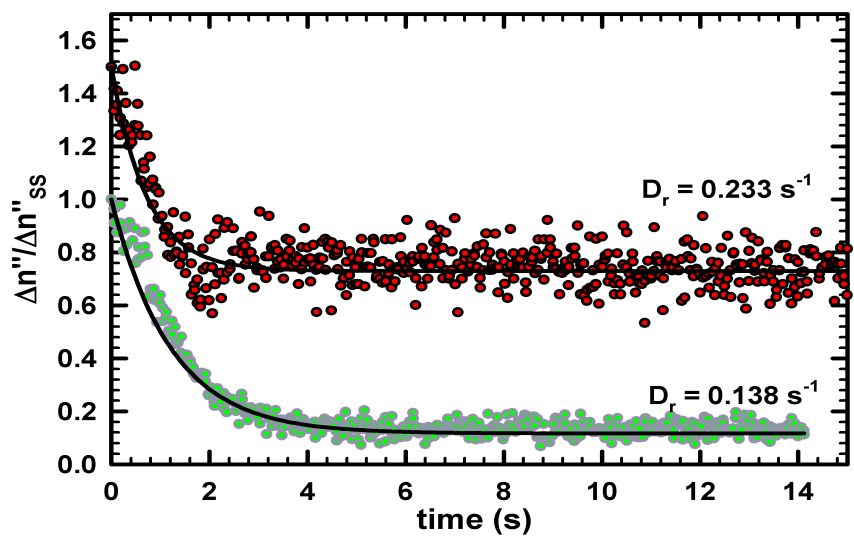

Figure 5: Transient dichroism normalised by the steady state value as a function of time, after cessation of flow in the high Peclet regime for oblate gold nanoparticles. Solid lines are single exponential fits with $\beta=1$ in Eq. 6. The data has been shifted for clarity, where the top data (red symbols) is for $170 \mathrm{~nm}$ and the bottom data (green) is for $292 \mathrm{~nm}$.

Dichroism relaxation upon flow cessation for FGS suspension are shown to be more gradual (Fig. 6). The average relaxation data (averaged over four concentrations, all in the dilute regime) is fitted using Eq. 4 with $\beta=0.61$. Unlike oblate gold spheroids, the small $\beta$ necessary to fit dichroism relaxation of FGS suspension indicates large polydispersity, as SLS experiments already revealed the sheets to be rather flat and not crumpled. The mean relaxation time is obtained using Eq. 6 and the corresponding rotational diffusion coefficient 
Dr $=0.04 \mathrm{~s}^{-1} \cdot{ }^{42}$

$$
<\tau>=\frac{\tau}{\beta} \Gamma\left\{\frac{1}{\beta}\right\}
$$

where $\Gamma$ is the gamma function. Using Eq. 7 for flat circular disk an average diameter of 900 $\mathrm{nm}$ is obtained from the mean relaxation time.

$$
d^{3}=\frac{3 k_{\mathrm{B}} T}{4 \eta_{o} D_{r}}
$$

here, $d$ is the diameter of the flat circular disk. The effective size of the FGS obtained by dichroism relaxation is slightly higher than the average size of 500-600 nm measured by AFM. ${ }^{38}$ The difference between the size obtained from dichroism and AFM can be attributed to the large polydispersity of the graphene sheets, relatively small statistics from AFM measurements, and the dominant contribution of larger particles to the scattering in flow dichroism.

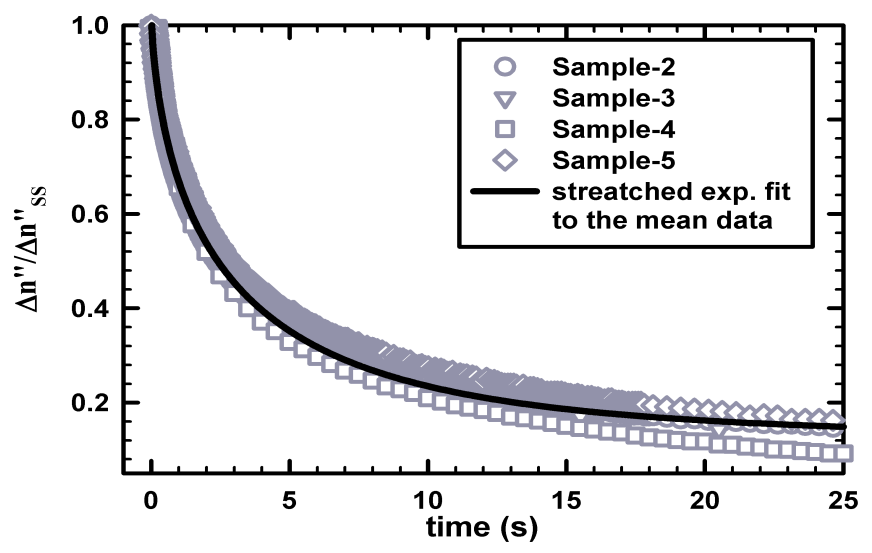

Figure 6: Transient dichroism normalised by steady state value as a function of time after cessation of flow in the hydrodynamic regime for four different concentrations of FGS suspensions. Solid line is a stretched single exponential fit to the average with $\beta=0.61$ in Eq. 6 . 


\section{Orientation of rigid Brownian particles}

We consider a dilute suspension of inertialess identical particles, and treat them as spheroids. The adjective dilute implying that each particle is hydrodynamically independent. Each particle has an aspect ratio $r(r>1$ or $<1$ for a prolate and oblate spheroid, respectively) and its orientation is defined by an unit vector $\mathbf{p}$ parallel to the axis of revolution of the spheroid. In these conditions, the time change of the single spheroid orientation follows the Jeffery's equation: ${ }^{36}$

$$
\dot{\mathbf{p}}=\Omega \cdot \mathbf{p}+\lambda(\mathbf{E} \cdot \mathbf{p}-\mathbf{E}: \mathbf{p p p})
$$

where $\mathbf{E}$ is the rate of strain tensor and $\boldsymbol{\Omega}$ the vorticity tensor. $\lambda$ represents the spheroid form factor and it is defined as the ratio $\left(r^{2}-1 / r^{2}+1\right)$. For very long thin rods, $\lambda$ is equal to 1 while for flat disks, it is equal to -1 . To describe the evolution of the orientation of a suspension of spheroids, it is necessary to employ the orientation distribution function $\psi(\mathbf{p}, t)$ defined such that the probability to find a particle oriented within a solid angle $d \mathbf{p}$ of $\mathbf{p}$ is $\psi d \mathbf{p}$. The time evolution of $\psi$ is governed by the following equation: ${ }^{45}$

$$
\frac{\partial \psi}{\partial t}+\nabla \cdot\left(\dot{\mathbf{p}} \psi-D_{r} \nabla \psi\right)=0
$$

where $\mathrm{D}_{r}$ is the rotational Brownian coefficient. The term in between the brackets represents the flux of the particle probability in the orientation space due to the torque induced by the external flow field and to the random effect of Brownian motion. At steady state the previous equation simplifies in

$$
\nabla \cdot\left(\dot{\mathbf{p}} \psi-D_{r} \nabla \psi\right)=0
$$

From the orientation distribution, it is possible to calculate the second order orientation 
tensor defined as:

$$
\mathbf{a}_{2}=\int \mathbf{p p} \psi d \mathbf{p}
$$

This tensor contains average information on the overall orientation state of the system. Following Fuller (1995), ${ }^{33}$ we can express the average orientation angle with respect to the flow direction as function of the orientation tensor as

$$
\chi=\frac{1}{2} \arctan \left(\frac{2 a_{12}}{a_{11}-a_{22}}\right)
$$

where $a_{i j}$ are the components of $\mathbf{a}_{2}$. We solve numerically eq. (10) since no simple analytical solution is possible. We employ a finite volume method as previously performed by Ferec et al. ${ }^{46,47}$ (see Ferec et $a l^{46}$ for more details about the mesh and the treatment of the periodic boundary conditions). A central scheme is implemented to interpolate properties between nodes and to obtain a physically steady state solution. Once the orientation distribution is numerically solved for a given shear rate, the orientation tensor is evaluated and the average orientation angle is consequentially obtained. In the model two input parameters are required: $\lambda$ and $\mathrm{D}_{r}$. These two parameters can be obtained experimentally from TEM and AFM particle characterisation and from the dichroism relaxation behaviour $\left(\mathrm{D}_{r}\right)$. In Table 2 , the values for the two parameters are reported for graphene and gold nanoparticles:

Table 2: Graphene and gold nanoparticles $\lambda$ and $\mathrm{D}_{r}$ used in the model

\begin{tabular}{lll}
\hline Particle & $\lambda$ & $\mathrm{D}_{r}$ \\
\hline Graphene & -1 & $0.04 \mathrm{~s}^{-1}$ \\
Gold-170nm & -0.53 & $0.233 \mathrm{~s}^{-1}$ \\
Gold-292nm & -0.53 & $0.138 \mathrm{~s}^{-1}$ \\
\hline
\end{tabular}

The orientation angle predicted by the model and the experimental results are compared in Figure 7. The model, without any fitting parameters, is able to overall predict the orientation dynamics of these two systems.

In the case of gold nanoparticles, the suspensions are slightly polydisperse (with a polydispersity index of $0.1^{19}$ ). The model predicts correctly the effect of the slightly lower $\mathrm{D}_{r}$ for 


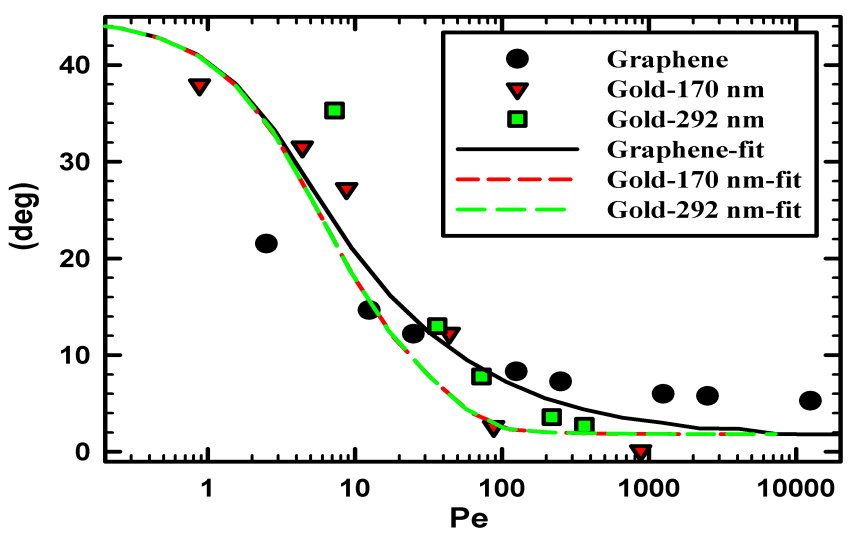

Figure 7: Comparison between experiments and theoretical predictions for average orientation angle as a function of Peclet number for FGS and oblate gold nanoparticles.

the system Gold-292nm. Lower $\mathrm{D}_{r}$ implies higher Peclet numbers $\left(\mathrm{Pe}=\dot{\gamma} / D_{r}\right)$ at equal shear rate applied. Hence the particles have lower average orientation angle at equal shear rate compared with the results (experimentally and in the model) for the system Gold-170nm. The differences between the model predictions and the experimental results can be due to approximation in the particle shape which in the model are assumed to be oblate spheroid (while in reality, they have a decahedral shape) and to neglecting the weak polydispersity. In the case of FGSs, the model shows larger deviations at low Pe and this deviation levels off increasing the Pe. It is important to underline that the graphene suspensions analysed here are very polydisperse while in the model, the particles are considered to be monodisperse and the shape may not be fully represented. In order to account for polydispersity, we defined subsets of the graphene suspensions and calculated (for each of the subset) the average $\bar{D}_{r}$ starting from the AFM characterisation showed in Fig. 1. Since the system is dilute, each subset of particles can be simulated independently from the rest of the subsets. Hence, the $N_{i}$ particles in the subset $i$ have an average rotational diffusion $\bar{D}_{r, i}$ (calculated according eq. (7)) and average diameter $\bar{D}_{i}$. By solving eq. (10) with $\bar{D}_{r, i}$, we can calculate the evolution of average orientation angle for the subset $i, \chi_{i}$. Once all the subsets are solved, we average the results for the orientation angles in two ways: a number $\bar{\chi}_{N}$ and a surface average $\bar{\chi}_{S}$ 


$$
\begin{gathered}
\bar{\chi}_{N}=\frac{\sum \chi_{i} N_{i}}{\sum N_{i}} \\
\bar{\chi}_{S}=\frac{\sum \chi_{i} N_{i} \bar{D}_{i}^{2}}{\sum N_{i} \bar{D}_{i}^{2}}
\end{gathered}
$$

We can observe in Fig. 8 that the surface average results (Eq. 14) are closer to the experimental values with respect to the model prediction obtained for monodisperse system and polydisperse using a number average (Eq. 13). This implies that the dichroism results are influenced more by the larger size particles in the case of a polydisperse system, which can be rationalised by the scattering power of the objects. This also explains the deviation at low Pe between the model predictions in the case of assuming the system monodisperse. Since the dichroic response is more influenced by larger particles, the Peclet numbers that we are probing experimentally are effectively higher than the one simulated (assuming an average rotational diffusion coefficient) and hence, the discrepancy at low shear rates. Finally, the good agreement between the model predictions assuming the shape of particles as thin disks and the experimental data confirms that the FGS studied here behaves as 2D structures with flat surfaces once suspended in the liquid medium.

\section{Conclusions}

In this paper, flow alignment of functionalized graphene sheets in dilute suspension was studied using rheo-optical measurements and compared to theoretical predictions and with results for solid gold decahedra. To describe their orientation dynamics, the FGS particles can be treated as solid flat sheets, despite the relatively low local bending modulus of the sheets. We observe in steady shear experiments the orientation angle of FGS with respect to flow direction decreased with a slope of -0.25 when plotted versus shear rate (or Pe number)

where as for gold nanoparticles showed a slope of -0.9. Relaxation experiments confirmed 


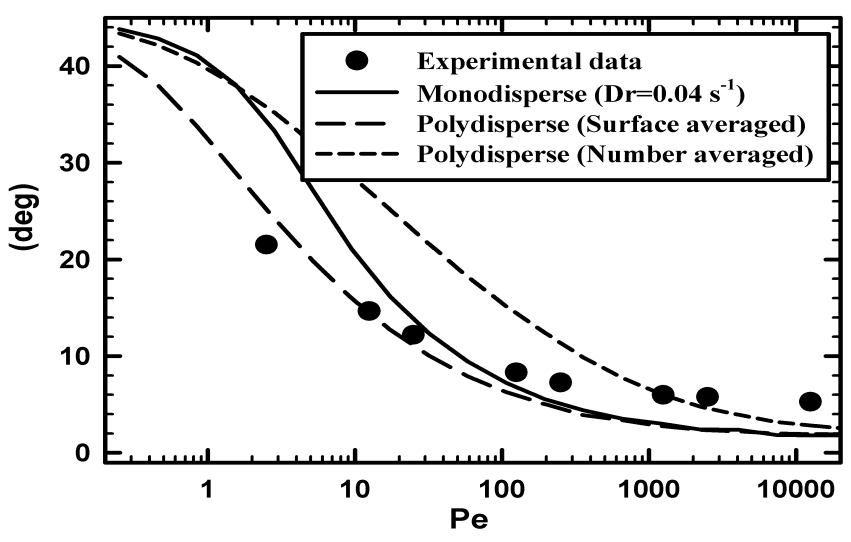

Figure 8: Comparison between experimental data and theoretical predictions for FGS. If surface averaged polydispersity is taken into account then the theoretical predictions are closer to the experimental data when compared to the predictions assuming monodispersity or number averaged polydispersity.

that this difference is due to a higher polydispersity. Moreover, in both cases, the results demonstrate that these particles behave as flat, rigid particles and that equivalent spheroids is a first order approximation to characterise the orientation of these systems. In the case of FGS, we reproduce the polydispersity of the system by simulating subset of particles with different average rotational diffusion. Interestingly, we found that the surface average polydispersity gives a better prediction to experimental results. This implies that the dichroism results (as a scattering technique) is influenced to a greater extent by larger particles rather than smaller one when the system is polydisperse. Quantitative agreement between dichroism experiments and model predictions indicates FGS to be made of flat surface without any crumpling. We also performed static light scattering measurements that showed FGS to have flat 2D surface in suspension without any indication of crumpling.

\section{References}

(1) Geim, A. K. Graphene: Status and Prospects. Science 2009, 324, 1530-1534. 
(2) Lee, C.; Wei, X.; Kysar, J. W.; Hone, J. Measurement of the Elastic Properties and Intrinsic Strength of Monolayer Graphene. Science 2008, 321, 385-388

(3) Balandin, A. A.; Ghosh, S.; Bao, W.; Calizo, I.; Teweldebrhan, D.; Miao, F.; Lau, C. N. Superior Thermal Conductivity of Single-Layer Graphene. Nano Lett. 2008, 8, $902-907$.

(4) Allen, M. J.; Tung, V. C.; Kaner, R. B. Honeycomb Carbon: A Review of Graphene. Chem. Rev. 2010, 110, 132-145.

(5) Kim, H.; Abdala, A. A.; Macosko, C. W. Graphene/Polymer Nanocomposites. Macromolecules 2010, 43, 6515-6530.

(6) Poulin, P.; Jalili, R.; Neri, W.; Nallet, F.; Divoux, T.; Colin, A.; Aboutalebi, S. H.; Wallace, G.; Zakri, C. Superflexibility of Graphene Oxide, Proc. Natl. Acad. Sci. 2016, 113, 11088-11093.

(7) Naficy, S.; Jalili, R.; Aboutalebi, S. H.; Gorkin, R. A.; Konstantinov, K.; Innis, P. C.; Spinks, G. M.; Poulin, P.; Wallace, G. G. Graphene Oxide Dispersions: Tuning Rheology to Enable Fabrication, Mater. Horiz. 2014, 1, 326-331.

(8) Liu, P.; Zhang, Y. W. Temperature-Dependent Bending Rigidity of Graphene. Appl. Phys. Lett. 2009, 94, 231912.

(9) Shim, J.; Yun J. M.; Yun, T.; Kim, P.; Lee, K. E.; Lee, W. J.; Ryoo, R.; Pine, D. J.; Yi, G.; Kim, S. O. Two-Minute Assembly of Pristine Large-Area Graphene Based Films, Nano Lett. 2014, 14, 1388-1393.

(10) Imperiali, L.; Liao, K.; Clasen, C.; Fransaer, J.; Macosko, C. W.; Vermant, J. Interfacial Rheology and Structure of Tiled Graphene Oxide Sheets, Langmuir 2012, 28, 79908000 . 
(11) Weber, C. M.; Eisele, D. M.; Rabe, J. P.; Liang, Y.; Feng, X.; Zhi, L.; Mullen, K.; Lyon, J. L.; Williams, R.; Vanden Bout, D. A.; Stevenson, K. J. Graphene-Based Optically Transparent Electrodes for Spectroelectrochemistry in the UV-Vis Region, Small, 2010, 6 184-189.

(12) Kang, X.; Wang, J.; Wu, H.; Liu, J.; Aksay, I. A.; Lin, Y. A graphene-Based Electrochemical Sensor for Sensitive Detection of Paracetamol, Talanta 2010, 81, 754-759.

(13) Okagawa, A.; Mason, S. G. The Kinetics of Flowing Dispersions. VII. Oscillatory Behavior of Rods and Discs in Shear Flow. J. Colloid. Interface. Sci. 1973 45, 330-358.

(14) Frattini, P. 1.; Fuller, G.G. The Dynamics of Dilute Colloidal Suspensions Subject to Time-Dependent Flow-Fields by Conservative Dichroism. J. Colloid. Interface. Sci. 1984, 100, 506-518.

(15) Frattini, P. l.; Fuller, G.G. Conservative Dichroism Of A Sheared Suspension In The Rayleigh-Gans Light-Scattering Approximation. J. Colloid. Interface. Sci. 1987, 119, 335-351.

(16) Johnson, S. J.; Frattini, P. L.; Fuller, G. G. Simultaneous Dichroism and Birefringence Measurements of Dilute Colloidal Suspensions in Transient Shear Flow. J. Colloid. Interface. Sci. 1985, 104, 440-455.

(17) Vermant J.; Yang H.; Fuller G.G. Rheooptical Determination of Aspect Ratio And Polydispersity of Nonspherical Particles. AIChE J. 2001, 47, 790-798.

(18) Gunes, D. Z.; Scirocco, R.; Mewis, J.; Vermant, J. Flow-Induced Orientation of Non-Spherical Particles: Effect of Aspect Ratio and Medium Rheology. J. Non-Newtonian Fluid Mech. 2008, 155, 39-50.

(19) Reddy, N. K.; Pérez-Juste, J.; Pastoriza-Santos, I.; Lang, P. R.; Dhont, J. K. G.; 
Liz-Marzán, L. M.; Vermant, J. Flow Dichroism as a Reliable Method to Measure the Hydrodynamic Aspect Ratio of Gold Nanoparticles. ACS Nano 2011, 5, 4935-4944.

(20) Natale, G.; Reddy, N. K.; Ausias, G.; Frec, J.; Heuzey M. C.; Carreau P. Rheo-Optical Response of Carbon Nanotube Suspensions. J. Rheol. 2015, 59, 499-524.

(21) Reinhardt, U. T.; Meyer de Groot, E. L.; Fuller, G. G.; Kulicke W.-M. RheoOptical Characterization (Flow-Birefringence and Flow-Dichroism) of the Tobacco Mosaic Virus. Macromol. Chem. Phys. 1995, 196, 63-74.

(22) Lenstra, T. A. J.; Dhont, J. K. G. Flow Dichroism in Critical Colloidal Fluids. Phys. Rev. E 2001, 63, 061401.

(23) Hilliou, L.; Vlassopoulos, D.; Rehahn, M. Dynamics of Hairy-Rod Polymer Solutions in Simple Shear Flow: Aging Effects. Macromolecules 2000, 33, 3105-3110.

(24) Hilliou, L.; Vlassopoulos, D.; Rehahn, M. Dynamics of Nondilute Hairy-Rod Polymer Solutions in Simple Shear Flow. Macromolecules 2001, 34, 1742-1750.

(25) Mobuchon, C.; Heuzey, M. C.; Carreau, P. J.; Reddy, N. K.; Vermant, J. Anisotropy of Non-Aqueous Layered Silicate Suspensions Subjected to Shear Flow. J. Rheol. 2009, $53,517-538$.

(26) Hwa, T.; Kokufuta, E.; Tanaka, T. Conformation of Graphite Oxide Membranes in Solution, Phys. Rev. A 1991 44, R2235.

(27) Wen, X.; Garland, C. W.; Hwa, T.; Kardar, M.; Kokufuta, E.; Li, Y.; Orkisz, M.; Tanaka, T. Crumpled and Collapsed conformations in Graphite Oxide Membranes. Nature 1992, 355, 426-428.

(28) Spector, M. S.; Naranjo, E.; Chiruvolu, S.; Zasadzinski, J. A. Conformation of Tethered Membrane: Crumpling in Graphite Oxide? Phys. Rev. Lett. 1994, 73, 2867. 
(29) Schlüter, A. D.; Payamyar, P.; and Öttinger, H. S. How the World Changes By Going from One -to Two- Dimensional Polymers in Solution. Macromol. Rapid Commun. 2016, 37, 1638-1650.

(30) Stankovich, S.; Dikin, D. A.; Dommett, G. H. B.; Kohlhaas, K. M.; Zimney, E. J.; Stach, E. A.; Piner, R. D.; Nguyen, S. T.; Ruoff, R. S. Graphene-Based Composite Materials. Nature, 2006, 442, 282-286.

(31) Fry, D.; Langhorst, B.; Kim, H.; Grulke, E.; Wang, H.; and Hobbie, E. K. Anisotropy of Sheared Carbon-Nanotube Suspensions. Phys. Rev. Lett. 2005, 95, 038304.

(32) Fry, D., Langhorst, B.; Wang, H.; Becker, M. L.; Bauer, B. J.; Grulke, E.; and Hobbie, E. K. Rheo-Optical Studies of Carbon Nanotube Suspensions. J. Chem. Phys. 2006, 124, 054703.

(33) Fuller, G. G. Optical Rheometry of Complex Fluids; Oxford University Press: Oxford, 1995.

(34) Doi, M.; Edwards, S. F. The Theory of Polymer Dynamics; Oxford University Press: Oxford, 1988.

(35) Bird, R. B.; Armstrong, R. C.; Hassager, O.; Curtiss, C. F.; Middleman, S. Dynamics of Polymeric Liquids; John Wiley and sons: New York, 1978, 2.

(36) Jeffery, G. B. The Motion of Ellipsoidal Particles Immersed in a Viscous Fluid. Proc. Royal Soc. A 1922, 102, 161-179.

(37) Bretherton, F. P. The Motion of Rigid Particles in a Shear Flow at Low Reynolds Number. J. Fluid Mech. 1962, 14, 284-304.

(38) McAllister, M. J.; Li, J. L.; Adamson, D. H.; Schniepp, H. C.; Abdala, A. A.; Liu, J.; Alonso, M. H.; Milius, D. L.; Car, R.; Prud'homme, R. K.; Aksay, I. A. Single 
Sheet Functionalized Graphene by Oxidation and Thermal Expansion of Graphite. Chem. Mater. 2007, 19, 4396-4404.

(39) Schniepp, H. C.; Li, J.; McAllister, M. J.; Sai, H.; Herrera-Alonso, M.; Adamson, D. H.; Prud?homme, R. K.; Car, R.; Saville, D. A.; Aksay, I. A. Functionalized Single Graphene Sheets Derived from Splitting Graphite Oxide. J. Phys. Chem. B 2006, 110, $8535-8539$.

(40) Pellens, L.; Vermant, J.; Mewis, J. Deviations from the Stress-Optical Relation in Telechelic Associative Polymer Solutions. Macromolecules 2005, 38, 1911-1981

(41) Maguire, J. F.; McTague, J. P.; Rondelez, F. Rotational Diffusion of Sterically Interacting Rodlike Macromolecules. Phys. Rev. Lett. 1980, 45, 1891.

(42) Lindsey, C. P.; Patterson, G. D. Detailed Comparison of the Williams-Watts and ColeDavidson Functions. J. Chem. Phys 1980, 73, 3348.

(43) Koenig, S. H. Brownian Motion of an Ellipsoid. A Correction to Perrin's Results. Biopolymers 1975, 14, 2421-2423.

(44) Berberan-Santos, M. N.; Bodunov, E. N.; Valeur, B. Mathematical Functions for the Analysis of Luminescence Decays with Underlying Distributions 1. Kohlrausch Decay Function (Stretched Exponential). Chem. Phys. 2005, 315, 171-182.

(45) Leal, L. G.; Hinch, E. J. The Rheology of a Suspension of Nearly Spherical Particles Subject to Brownian Rotations. J. Fluid Mech., 1972, 55, 745-765.

(46) Férec, J.; Heniche, M.; Heuzey, M. C.; Ausias, G.; Carreau, P. J. Numerical Solution of the Fokker-Planck Equation for Fiber Suspensions: Application to the Folgar-TuckerLipscomb Model. J. Non-Newtonian Fluid Mech. 2008, 155, 20-29. 
(47) Natale, G., Heuzey, M. C., Carreau, P. J., Ausias, G., and Frec, J., Rheological Modeling of Carbon Nanotube Suspensions with Rod-Rod Interactions. AIChE J. 2014, 60, 1476-1487.

\section{Acknowledgement}

The authors thank Luiz Lis-Marzán, Jorge Pérez-Juste and Isabel Pastoriza-Santos for providing oblate gold spheroids. JV acknowledges the Swiss National Science Foundation, project number 200021-157147 and GN acknowledges funding from the NSERC Grant No. RGPIN-2017-03783.

\section{List of changes}




\section{Graphical TOC Entry}

Some journals require a graphical entry for the Table of Contents. This should be laid out "print ready" so that the sizing of the text is correct. Inside the tocentry environment, the font used is Helvetica $8 \mathrm{pt}$, as required by Journal of the American Chemical Society.

The surrounding frame is $9 \mathrm{~cm}$ by $3.5 \mathrm{~cm}$, which is the maximum permitted for Journal of the American Chemical Society graphical table of content entries. The box will not resize if the content is too big: instead it will overflow the edge of the box.

This box and the associated title will always be printed on a separate page at the end of the document. 